\title{
Outcomes of surgery in the treatment of isolated nonnative mitral valve infective endocarditis
}

\author{
Kevin L. Greason, MD, ${ }^{\mathrm{a}}$ Mathew Thomas, MD, ${ }^{\mathrm{a}}$ James M. Steckelberg, MD, ${ }^{\mathrm{b}}$ Richard C. Daly, MD, \\ Hartzell V. Schaff, MD, ${ }^{a}$ Zhuo Li, MS, ${ }^{c}$ and Joseph A. Dearani, MD ${ }^{\mathrm{a}}$
}

Objective: We reviewed our experience with the operative management of patients with isolated nonnative mitral valve infective endocarditis to better understand the outcome.

\begin{abstract}
Methods: We reviewed the records of 39 patients operated on for isolated nonnative mitral valve infective endocarditis from January 1974 to June 2009. Median age of the group was 68 years. There were $23(59 \%)$ women. Prostheses were mechanical in $18(46 \%)$ patients, biological in $18(46 \%)$, and annuloplasty rings in $3(8 \%)$. Staphylococcus was present in $22(56 \%)$ patients. Operative indications included valve dysfunction in 26 $(67 \%)$ patients and heart failure in $22(56 \%)$.

Results: Perivalvular abscess was present in $12(31 \%)$ patients. Replacement valves were mechanical in 23 $(59 \%)$ patients and biological in $16(41 \%)$. Twenty $(51 \%)$ patients received additional operative procedures. Treatment-related mortality occurred in $8(21 \%)$ patients, with age being the only factor predictive of mortality (hazard ratio, 5.37). Follow-up of the survivors was 5.7 years. Six (18\%) patients underwent repeat mitral valve replacement including 3 who had an annulus abscess at the initial operation and 2 who had the prosthesis sutured to the left atrial wall. There was $1(4 \%)$ case of recurrent endocarditis in the group of 28 patients who survived more than 1 year after the incident operation. Survival at 5 years was $48 \%(95 \%$ confidence interval, $35 \%-67 \%$ ).
\end{abstract}

Conclusions: Surgery for isolated nonnative mitral valve infective endocarditis carries increased operative risk. Aggressive debridement and reconstruction of the annulus are paramount to achieving a good outcome. Surviving patients obtain high rates of cure and freedom from recurrent infective endocarditis. (J Thorac Cardiovasc Surg 2014;147:349-54)

The purpose of this article is to study and report outcomes of patients operated on for isolated nonnative mitral valve infective endocarditis. Infection of the nonnative mitral valve is a serious condition associated with significant morbidity and mortality. Fortunately, it is an uncommon problem, with a reported incidence of less than $0.5 \%$ per patient-year. ${ }^{1}$ Owing to its rarity, there are few studies that have specifically studied the condition. Most surgical series report outcomes of either mitral valve (native and nonnative) or nonnative valve (aortic, mitral, or combined) infective endocarditis, but not specifically the outcomes of isolated nonnative mitral valve infective endocarditis. ${ }^{2-4}$ Still, these same studies identify worse outcomes in patients with nonnative over native valve and mitral over aortic valve infective endocarditis. ${ }^{2-4}$ This article focuses on the select

\footnotetext{
$\overline{\text { From the Divisions of Cardiovascular Surgery }}{ }^{\mathrm{a}}$ and Infectious Diseases ${ }^{\mathrm{b}}$ and the Department of Biostatistics/Cardiovascular Surgery, ${ }^{\mathrm{c}}$ Mayo Clinic, Rochester, Minn. Disclosures: Authors have nothing to disclose with regard to commercial support. Received for publication Aug 7, 2012; revisions received Nov 7, 2012; accepted for publication Dec 5, 2012; available ahead of print Jan 14, 2013

Address for reprints: Kevin L. Greason, MD, Division of Cardiovascular Surgery, Mayo Clinic, 200 First St, SW, Rochester, MN 55905 (E-mail: greason.kevin@ mayo.edu).

$0022-5223 / \$ 36.00$

Copyright (C) 2014 by The American Association for Thoracic Surgery

http://dx.doi.org/10.1016/j.jtcvs.2012.12.007
}

group of patients with isolated nonnative mitral valve infective endocarditis.

\section{PATIENTS AND METHODS}

Mayo Clinic Institutional Review Board approved the collection and analysis of data for this study. We reviewed the prospectively collected databases of the Divisions of Infectious Disease and Cardiovascular Surgery, Mayo Clinic, Rochester, Minnesota, to identify all patients treated for isolated nonnative mitral valve infective endocarditis. We defined nonnative mitral valve infective endocarditis as infection based on standard bacteriologic blood and tissue cultures of a mitral valve prosthetic ring or valve. In the case of negative cultures, we did not perform additional testing such as genomic sequencing. We excluded patients who had previous other valve repair or replacement.

Between January 1, 1974, and June 1, 2009, 94 patients received treatment for isolated nonnative mitral valve infective endocarditis. Sixty-five $(63 \%)$ patients received isolated medical therapy, and $39(37 \%)$ patients received mitral valve replacement in addition to medical therapy. These 39 patients who received operation form the basis for this study.

We reviewed each operative patient's medical record. We collected data about patient characteristics, operative findings and techniques, operative morbidity and mortality, and follow-up outcomes to include recurrence of endocarditis, need for subsequent mitral valve operation, and survival. We queried the Social Security Death Index to complete collection of survival data

For the purposes of this study, we used the following definitions: operative mortality occurred within 30 days of the incident operation or during the same hospital stay; treatment-related mortality occurred greater than 30 days from the operation and after hospital discharge related to the incident infective endocarditis episode. Last, cure of infective endocarditis was defined as microbiologic cure evidenced by negative blood cultures off 
antibiotics and the absence of a relapse of endocarditis at the follow-up examination/visit.

Descriptive statistics for categorical variables reported are frequency and percentage; continuous variables reported are median (range). We compared categorical variables with the Fisher's exact test and continuous variables with the 2-sample $t$ test or Wilcoxon rank sum test, where appropriate. We used logistic regression models to identify univariate and multivariate predictors of perioperative mortality. We report survival of patients and survival as determined by type of prosthesis inserted using Kaplan-Meier survival curves. All statistical tests were 2-sided.

\section{RESULTS}

We report baseline patient characteristics of the 39 patients in Table 1. Previous infective endocarditis occurred in 15 $(38 \%)$ patients and involved the native mitral valve in 5 (33\%) patients and the nonnative mitral valve in $10(67 \%)$. Additional preoperative findings included a classification of early infective endocarditis ( $\leq 12$ months after valve operation) in $12(31 \%)$ patients and late infective endocarditis (>12 months after valve operation) in 27 (69\%). Medical therapy included culture-directed antibiotic treatment for 46 days (5-222 days) and postoperative for 28 days (1-181 days).

Staphylococci were the most common causative bacteria (22 patients, 56\%). Specific isolates included methicillinsensitive Staphylococcus aureus in $10(26 \%)$ patients, coagulase-negative staphylococci in $9(23 \%)$, methicillinresistant $S$ aureus in $2(5 \%)$, and a mixed infection owing to coagulase-negative Staphylococcus and Streptococcus in $1(3 \%)$. The remaining $17(44 \%)$ nonstaphylococcal bacterial causes included Enterococcus in $5(13 \%)$ patients, viridans group streptococci in $5(13 \%)$, and negative cultures in 3 ( $8 \%$, occurring in 1995, 2002, and 2005), Propionibacterium acnes in 2 (5\%), Haemophilis parainfluenza in $1(3 \%)$, and the fungus Homographiella aspergillata in $1(3 \%)$.

Infection included the following specific isolates in the group of 12 patients with early infective endocarditis.

TABLE 1. Patient characteristics

\begin{tabular}{lc}
\hline \multicolumn{1}{c}{ Variable } & Data \\
\hline Age $(y)$ & $68.0(15.8-82.3)$ \\
Patients $\geq 65$ y & $26(67 \%)$ \\
Female gender & $23(59 \%)$ \\
No. of previous sternotomies & \\
One & $27(69 \%)$ \\
Two & $9(23 \%)$ \\
Three & $3(8 \%)$ \\
Prosthesis type & \\
Mechanical & $18(46 \%)$ \\
Biological & $18(46 \%)$ \\
Ring annuloplasty & $3(8 \%)$ \\
Preoperative ejection fraction & \\
$\geq 50 \%$ & $30(77 \%)$ \\
$<50 \%$ & $8(21 \%)$ \\
Unknown & $1(3 \%)$ \\
Creatinine $\geq 1.8$ mg/dL & $6(15 \%)$ \\
\hline
\end{tabular}

The most common isolate was coagulase-negative staphylococci in $6(50 \%)$ patients. The remaining isolates included $S$ aureus in $2(17 \%)$ patients and Enterococcus, $H$ parainfluenza, $H$ aspergillata, and negative culture in 1 each.

The reported indication(s) for operation included valve dysfunction in $26(67 \%)$ patients, heart failure in 22 $(56 \%)$, embolism in $7(18 \%)$, sepsis in $6(15 \%)$, and hemolysis in $1(3 \%) ; 21(54 \%)$ had more than 1 indication. Operative status was elective in $23(59 \%)$ patients, urgent in 15 $(38 \%)$, and emergency in $1(3 \%)$. The median time from diagnosis of infective endocarditis to operation was 11 days (range, 0-259 days).

Operation included a cardiopulmonary bypass time of 113 minutes (59-207 minutes) and aortic crossclamp time of 67 minutes (0-145 minutes). Perfusion temperature was $32^{\circ} \mathrm{C}$ or more in 18 patients $(46 \%), 28^{\circ} \mathrm{C}$ or more and less than $32^{\circ} \mathrm{C}$ in $6(15 \%)$, and less than $28^{\circ} \mathrm{C}$ in 15 $(38 \%)$. Myocardial protection included isolated antegrade cardioplegia in $28(72 \%)$ patients and combined antegrade and retrograde cardioplegia in $9(23 \%)$. In $1(3 \%)$ patient, the surgeon used intermittent aortic crossclamp with cold fibrillatory arrest. In another patient, in whom it was the fourth mitral valve operation, the surgeon could not safely control the aorta such that he replaced the mitral valve during a 55-minute period of deep hypothermic circulatory arrest.

Technical aspects of the mitral valve operation included exposure of the mitral valve via a posterior interatrial groove incision in $25(64 \%)$ patients, a transseptal incision in $13(33 \%)$, and a dome of the left atrium incision in 1 $(3 \%)$. There was gross evidence of active infection with tissue destruction or vegetation in $34(87 \%)$ patients. There was no abscess cavity reported in $27(69 \%)$ patients, an abscess cavity that involved less than $50 \%$ of the circumference of the mitral valve annulus in $10(26 \%)$, and an abscess cavity that involved $50 \%$ or more of the circumference of the annulus in $2(5 \%)$. One $(3 \%)$ patient had abscess cavity involvement of the aortomitral intervalvular fibrosa that involved less than $50 \%$ of the circumference of the mitral valve annulus There were $14(36 \%)$ patients in whom the mitral valve demonstrated dehiscence from the annulus.

Operation included the removal of all infected material from the mitral valve annulus. In addition, 20 (51\%) patients received treatment of the annulus with a $10 \%$ phenol solution as part of our local institutional practice. There was $1(3 \%)$ patient with less than $50 \%$ abscess cavity involvement of the circumference of the mitral valve annulus who received pericardial patch reconstruction of the annulus before replacement of the new prosthesis. In $2(5 \%)$ patients, the prosthesis was sutured to the left atrial wall because of destruction of the mitral valve annulus. The remaining $36(92 \%)$ patients received standard suture 
placement and suturing of the new prosthesis. Valve replacement included a mechanical prosthesis in $23(59 \%)$ patients and a biological prosthesis in $16(41 \%)$. Twenty $(51 \%)$ patients received a concomitant operative procedure (Table 2).

Operative mortality occurred in $5(13 \%)$ patients. The patient who underwent replacement of the mitral valve during a period of hypothermic circulatory arrest died of multiple acute cerebral infarctions within the watershed area of the brain consistent with hypoperfusion phenomena. Additional causes of mortality included stroke, cardiac arrest from bradycardia, myocardial infarction, and multisystem organ failure in 1 each.

Patients who died had the following characteristics. Infections include $S$ aureus in 2 patients, methicillinresistant $S$ aureus in 1, coagulase-negative staphylococci in 1 , and negative culture in 1 . In 2 patients there was an annular abscess (both had $S$ aureus). Operative status was urgent/ emergency in 4 patients and elective in 1.

We report univariate predictors of operative mortality in Table 3. Importantly, staphylococcal infection $(P=.941)$, presence of an annulus abscess $(P=.512)$, and operative status $(P=.322)$ did not predict operative mortality. Age was the only significant factor and after adjusting for it with multivariate analysis (hazard ratio, 5.37; confidence interval, 2.09-13.77; $P<.001$ ), nothing else turned out to be significant.

Additional mortality occurred in $6(15 \%)$ patients within 1 year of operation. Importantly, 3 patients died of endocarditis-related complications that included sepsis, mediastinitis, and recurrent endocarditis requiring repeat mitral valve replacement. One of the remaining 3 deaths was the result of cardioembolic stroke, and the other 2 deaths were the result of unknown causes. One patient was lost to follow-up 6 days after operation but was alive at 10.5 years after operation as indicated by query of the Social Security Death Index. Median follow-up of the survivors was 5.7 years (range, 0.6-24.0).

Subsequent mitral valve replacement occurred in 6 $(18 \%)$ of the 34 patients who survived the operative

TABLE 2. Concomitant procedures performed at time of mitral valve replacement

\begin{tabular}{lc}
\hline \multicolumn{1}{c}{ Variable } & Data \\
\hline Any additional procedure & $20(51 \%)$ \\
Tricuspid valve repair & $10(26 \%)$ \\
Suture closure of left atrial appendage & $2(5 \%)$ \\
Aortic valve replacement (done for aortic stenosis) & $2(5 \%)$ \\
Coronary artery bypass graft surgery & $2(5 \%)$ \\
Tricuspid valve replacement & $1(3 \%)$ \\
Ventricular aneurysm repair & $1(3 \%)$ \\
Repair of left ventricle/left atrial appendage fistula & $1(3 \%)$ \\
Femoral pseudoaneurysm repair & $1(3 \%)$ \\
Femoral artery replacement & $1(3 \%)$ \\
\hline
\end{tabular}

TABLE 3. Univariate analysis of factors associated with operative mortality

\begin{tabular}{lccc}
\hline \multicolumn{1}{c}{ Parameter } & Hazard ratio & CI & $\boldsymbol{P}$ value \\
\hline Age $\geq 65$ y & 5.37 & $2.09-13.77$ & $<.001$ \\
Operative indication & & & \\
$\quad$ Heart failure & 1.72 & $0.81-3.64$ & .156 \\
$\quad$ Valve dysfunction & 1.45 & $0.67-3.11$ & .343 \\
$\quad$ Sepsis & 0.84 & $0.29-3.64$ & .155 \\
Status urgent/emergency & 1.44 & $0.74-2.14$ & .322 \\
Creatinine $>1.8$ mg/dL & 1.61 & $0.97-2.68$ & .064 \\
Ejection fraction $\geq 50 \%$ & 1.52 & $0.56-4.09$ & .411 \\
Operative status urgent/emergency & 1.44 & $0.7-2.98$ & .322 \\
Mitral valve annulus abscess & & & \\
$\quad \leq 50 \%$ of annulus & 1.32 & $0.58-3.0$ & .512 \\
Active endocarditis* & 1.12 & $0.39-3.23$ & .835 \\
CPB time (min) & 1.01 & $1-1.02$ & .114 \\
ACC time (min) & 1.01 & $0.99-1.02$ & .244 \\
Staphylococcal organism & 0.97 & $0.46-2.07$ & .941 \\
Valvular dehiscence & 0.86 & $0.41-1.81$ & .686 \\
Type of mitral valve replacement & 0.74 & $0.36-1.53$ & .421 \\
Days from diagnosis to operation & & & \\
$\quad \geq 14$ d & 0.73 & $0.35-1.51$ & .399 \\
$\quad \geq 7$ d & 0.71 & $0.34-1.48$ & .355 \\
\hline Cl Cong & & &
\end{tabular}

$C I$, Confidence interval; $C P B$, cardiopulmonary bypass; $A C C$, aortic crossclamp. *Defined by surgeon based on operative findings.

period and included 4 who had a mechanical prosthesis and 2 a biological prosthesis. One patient received repeat operation for acute bacterial endocarditis. In 3 patients the indication for repeat operation included severe paravalvular regurgitation not associated with infection. Both patients who had the prosthesis sutured to the left atrial wall had severe paravalvular leak develop and received subsequent mitral valve replacement, 1 at 60 days (patient had a $S$ aureus abscess at initial operation) and the other at 7.2 years after the index endocarditis operation. Of the 2 patients who did not have paravalvular leak/valvular dehiscence, 1 received replacement of a dysfunctional Ionescu-Shiley valve, and 1 received replacement because of a stuck leaflet that occurred after resection of a subaortic membrane.

Two patients had recurrent infective endocarditis and underwent repeat mitral valve replacement. Both patients initially had late nonnative valve $S$ aureus infective endocarditis and both had an annulus abscess at their initial operation. One patient had a relapse of $S a u$ reus infective endocarditis 34 days after the initial operation. Subsequent mitral valve replacement proved very difficult and the patient died of congestive heart failure 4 months after the repeat operation. A second patient received successful medical treatment of recurrent isolated nonnative mitral valve viridans group streptococci infective endocarditis 4.3 years after the incident endocarditis operation. He subsequently had severe paravalvular regurgitation develop and died during reoperation 
4.8 years after the medically treated episode of infective endocarditis.

Overall outcomes included operative mortality in 5 patients, further treatment-related mortality in 3, repeat mitral valve replacement in $6(18 \%)$ patients, and recurrence of infective endocarditis in $2(6 \%)$. Of those patients who survived more than 1 year after operation, cure of the endocarditis occurred in 27 of $28(96 \%)$ patients. Treatment of the annulus with phenol did not reduce the frequency of the combined end point of repeat mitral valve replacement, recurrent infective endocarditis, or infective endocarditisrelated death $(P=.52)$.

At last follow-up, 7 (18\%) patients were alive, including the patient lost to clinical follow-up (based on review of the Social Security Death Index). Survival at 5 years was $48 \%$ (95\% confidence interval, 35\%-67\%). The figures show the Kaplan-Meier estimated survival curves for operated patients and age- and gender-matched controls (Figure 1). In addition, we report survival based on type of valve prosthesis inserted (Figure 2).

\section{COMMENTS}

The present study describes our institution's 35-year operative experience in the treatment of patients with isolated nonnative mitral valve endocarditis and the associated outcomes of recurrent infective endocarditis, repeat mitral valve operation, and survival. Most literature on the treatment of patients with nonnative mitral valve infective endocarditis combines this group of patients with others such as those with native mitral valve or nonnative aortic valve endocarditis. ${ }^{2,3}$ And in most of these series, the patients with a nonnative mitral valve make up the minority of the cases in the study. The present discussion focuses on the group of patients with isolated nonnative mitral valve infective endocarditis.

We found that the indications for operation in our series were similar to those reported in other series and that sepsis

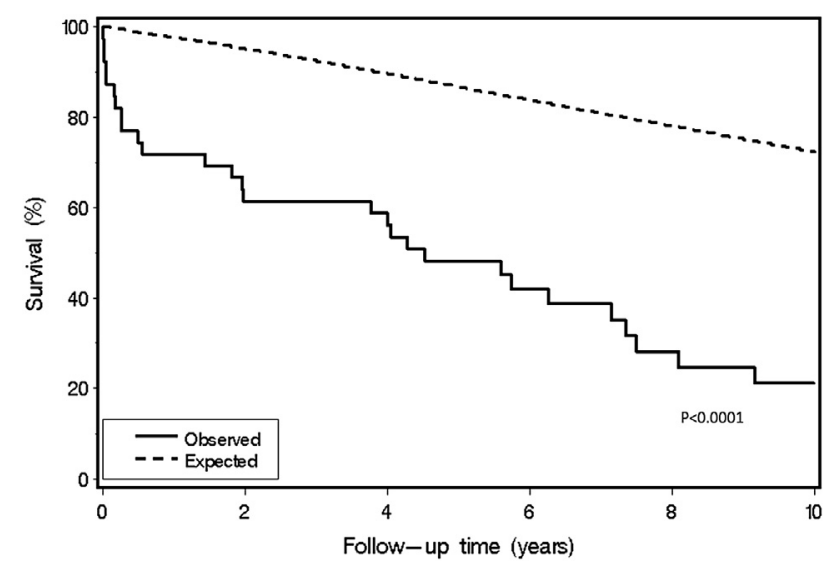

FIGURE 1. Kaplan-Meier estimated survival for operated patients with age- and gender-matched controls.

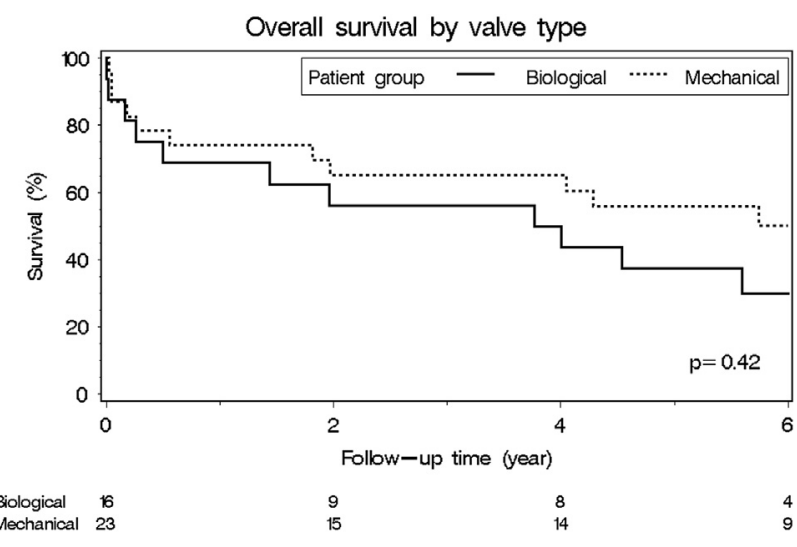

FIGURE 2. Kaplan-Meier estimated survival based on type of valve prosthesis inserted.

was an infrequent indication for operation. The majority of our patients $(54 \%)$ had more than 1 operative indication including valve dysfunction $(67 \%)$, heart failure $(56 \%)$, embolism $(18 \%)$, or sepsis $(15 \%)$. These operative indications are similar to those in the report by Musci and colleagues, ${ }^{2}$ in which heart failure was an indication in $74 \%$ of patients, with sepsis an indication in only $18 \%$. The low rate of sepsis as an operative indication may represent a vague definition of sepsis or a selection bias on the part of the surgeon, as Hill and colleagues ${ }^{5}$ showed that patients with sepsis are at higher risk of death with operation. It is possible that patients with sepsis received initial medical management and only after control of the sepsis were they taken to the operating room.

Most of the patients in our series received only standard operative techniques of hypothermic cardiopulmonary bypass, cardioplegic arrest of the heart, and exposure of the mitral valve through an incision posterior to the interatrial groove or through the atrial septum. Through that exposure, the surgeon removed all infected material, debrided the mitral valve annulus, and in most of the patients $(92 \%)$ used only standard mitral valve replacement techniques to suture in the prosthetic valve. Our aortic crossclamp time was 67 minutes, and that is not much different from that reported by Exposito and colleagues ${ }^{6}$ ( 63 minutes) in their series of 369 repeat mitral valve replacements for mostly non-endocarditis related indications.

We were probably not aggressive enough with mitral annulus debridement and reconstruction. An annulus abscess cavity was present in $12(31 \%)$ of our patients, and that is more frequent than the incidence reported by Musci and colleagues, ${ }^{2}$ who reported an abscess in only $5 \%$ of patients. Importantly, we had 4 patients with an abscess cavity, or with severe annulus destruction such that the surgeon sutured the prosthesis to the left atrial wall, in whom recurrent endocarditis or severe paravalvular regurgitation developed, necessitating repeat mitral valve operation. On the basis of this experience, we endorse 
the technique of aggressive annular debridement and pericardial patch reconstruction of the mitral valve annulus as needed before valve replacement as outlined by David and colleagues. ${ }^{7-9}$

Recurrent valve infection remains a problem for patients treated for nonnative mitral valve infective endocarditis. In our series, infective endocarditis recurred in $2(5 \%)$ patients and both had a $S$ aureus infection and an annulus abscess at the initial operation. It is possible that more aggressive debridement, such as described above, could have prevented recurrent infection. One patient died after repeat mitral valve operation and 1 survived after medical treatment. Sheikh and colleagues ${ }^{10}$ reported a similar frequency of recurrence of infective endocarditis in their series from the Toronto General Hospital (9/87 mitral valve replacement patients, $10 \%$ ). Importantly, in that series, 2 patients underwent reoperation and both survived, whereas 7 patients received medical management and only $1(14 \%)$ survived. There appears to be a low, but important, risk of recurrent infection in this patient group; should infection recur, treatment should be individualized, inasmuch as survival has been reported with both medical and surgical techniques.

There is risk of need for repeat mitral valve replacement in patients treated with operation for isolated nonnative mitral valve infective endocarditis. In our series, $6(18 \%)$ of the 34 patients who survived the initial operation underwent repeat mitral valve replacement. We expect that we could have reduced the need for repeat mitral valve replacement had we been more aggressive with debridement and annulus reconstruction, as previously noted. Regardless, our experience is similar to that of Exposito and colleagues, ${ }^{6}$ who reported the need for third time mitral valve replacement in 80 $(22 \%)$ of 369 patients. Importantly, in that study, only 5\% of the patients who needed the second mitral valve replacement had endocarditis; the remaining $95 \%$ of patients did not have endocarditis and presumably represented a less risky group of patients for valve replacement. However, Exposito's group reported a statistically significant relationship between the need for reoperation and annular disease that included endocarditis.

Urgent or emergency status is common in patients operated on for nonnative mitral valve infective endocarditis. In our series, $41 \%$ of patients underwent operation in a nonelective status, and that is similar to the $49 \%$ reported in the study by Sheikh and associates ${ }^{10}$ from the Toronto General Hospital group. Despite the frequent urgent nature of the operation, we report operative mortality in only 5 $(12.5 \%)$ of our patients; that is similar to the $8.7 \%$ reported by Sheikh and associates, ${ }^{10}$ but less than the $22.9 \%$ reported by Wang, Athan, and Pappas ${ }^{11}$ in the International Collaborative Study and the $32.8 \%$ reported by Musci and colleagues. ${ }^{2}$ In our study, operative status did not predict operative morality $(P=.322)$.
Operative mortality may not be an appropriate time frame to evaluate the mortality rate and clinical outcome of surgery in infective endocarditis, as pointed out by Musci and coworkers. ${ }^{3}$ In that regard, we noted 3 additional endocarditis-related deaths within 1 year of operation, and an endocarditis-related death occurred in $21 \%$ of our patients (8/39). Musci and associates ${ }^{2}$ also reported a higher 1-year mortality $(49 \%)$, but they did not report the endocarditis-related mortality. Multivariate analysis in our study identified age as the only factor significantly associated with survival, whereas Musci and coworkers ${ }^{2}$ reported nonelective operative status, preoperative renal insufficiency (not otherwise defined), and cardiogenic shock (intubated on arrival to the hospital) placed patients at higher risk of death.

Survival after operation is not affected by valve prosthesis type. In our series, the 1- and 5-year survival rates after biological valve implantation were $69 \% \pm 23 \%$ and $38 \% \pm 25 \%$; after mechanical valve implantation they were $74 \% \pm 18 \%$ and $56 \% \pm 21 \%$, the differences of which were not significant. In a like manner, Musci and associates $^{2}$ reported that comparison of survival rates after the implantation of biological or mechanical prostheses showed no differences in the groups out to 10 years. Moon and colleagues, ${ }^{12}$ from the Stanford University group, reported similar survival rate findings; however, long-term freedom from reoperation for patients less than 60 years who received a biological prosthesis was low, around $50 \%$ at 10 years. On the basis of these data, we recommend mechanical valve replacement to most patients under the age of 60 years.

There are 3 main limitations to this study: small number of patients, extensive time period of study, and retrospective design. These limitations are related to the rare occurrence of this condition, which makes it unlikely that any single institution could gather a large enough number of cases over a reasonable period of time in which to report pertinent outcomes. It would only be through a prospective multiinstitution registry, such as is available with aortic dissection and pericarditis, that a large enough experience could be garnered. Of these limitations, the extensive time period of study probably affects the results the most. We would expect, however, that just as operative mortality for other cardiac conditions has decreased over time, such would be the case for operation in the setting of nonnative mitral valve infective endocarditis, too; although, we cannot measure or analyze such an occurrence given the rarity of the condition.

Nonnative mitral valve infective endocarditis is a rare but serious condition associated with reduced survival. It remains a surgical challenge complicated by frequent presence of paravalvular abscess, need for annulus reconstruction, and need for concomitant operative procedure, all of which make an already difficult situation more complex. Aggressive mitral valve annulus debridement and pericardial patch reconstruction are paramount to a successful 
outcome. Unfortunately, treatment-related morbidity and mortality remain high and intermediate-term survival rates are less than in age- and gender-matched controls. However, freedom from recurrent infection in patients who survive the incident infective event is over $95 \%$, and we believe that is the basis for an aggressive surgical approach.

\section{References}

1. Arvay A, Lengyel M. Incidence and risk factors of prosthetic valve endocarditis. Eur J Cardiothorac Surg. 1988;2:340-6.

2. Musci M, Hubler M, Amiri A, Stein J, Kosky S, Meyer R, et al. Surgical treatment for active infective prosthetic valve endocarditis: 22-year single-center experience. Eur J Cardiothorac Surg. 2010;38:528-38.

3. Musci M, Hubler M, Pasic M, Amiri A, Stein J, Siniawski H, et al. Surgery for active infective mitral valve endocarditis: a 20 -year, single-center experience. J Heart Valve Dis. 2010;19:206-14.

4. Manne MB, Shrestha NK, Lytle BW, Nowicki ER, Blackstone E, Gordon SM, et al. Outcomes after surgical treatment of native and prosthetic valve infective endocarditis. Ann Thorac Surg. 2012;93:489-94.
5. Hill EE, Herregods MC, Vandershueren S, Claus P, Peetermans WE, Herijgers P. Management of prosthetic valve infective endocarditis. Am J Cardiol. 2008;101: 1174-8.

6. Exposito V, Garcia-Camarero T, Bernal JM, Amaiz E, Sarralde A, Garcia I, et al. Repeat mitral valve replacement: 30-years' experience. Rev Esp Cardiol. 2009; 62:929-32.

7. David TE, Regesta T, Gavra G, Armstrong S, Maganti MD. Surgical treatment of paravalvular abscess: long-term results. Eur J Cardiothorac Surg. 2007;31:43-8.

8. David TE, Gavra G, Feindel CM, Regesta T, Armstrong S, Maganti MD. Surgical treatment of active infective endocarditis: a continued challenge. J Thorac Cardiovasc Surg. 2007;133:144-9.

9. David TE, Feindel CM, Armstrong S, Sun Z. Reconstruction of the mitral anulus. J Thorac Cardiovasc Surg. 1995;110:1323-32.

10. Sheikh AM, Elhenawy AM, Maganti M, Armstrong S, David TE, Feindel CM. Outcomes of surgical intervention for isolated active mitral valve endocarditis. J Thorac Cardiovasc Surg. 2009;137:110-6.

11. Wang A, Athan E, Pappas PA. Contemporary clinical profile and outcome of prosthetic valve endocarditis. JAMA. 2007;297:1354-61.

12. Moon MR, Miller DC, Moore KA, Oyer PE, Mitchell RS, Robbins RC, et al. Treatment of endocarditis with valve replacement: the question of tissue versus mechanical prosthesis. Ann Thorac Surg. 2001;71:1164-71. 